\title{
Personalia
}

\section{0-th birthday of Christian von Ferber}

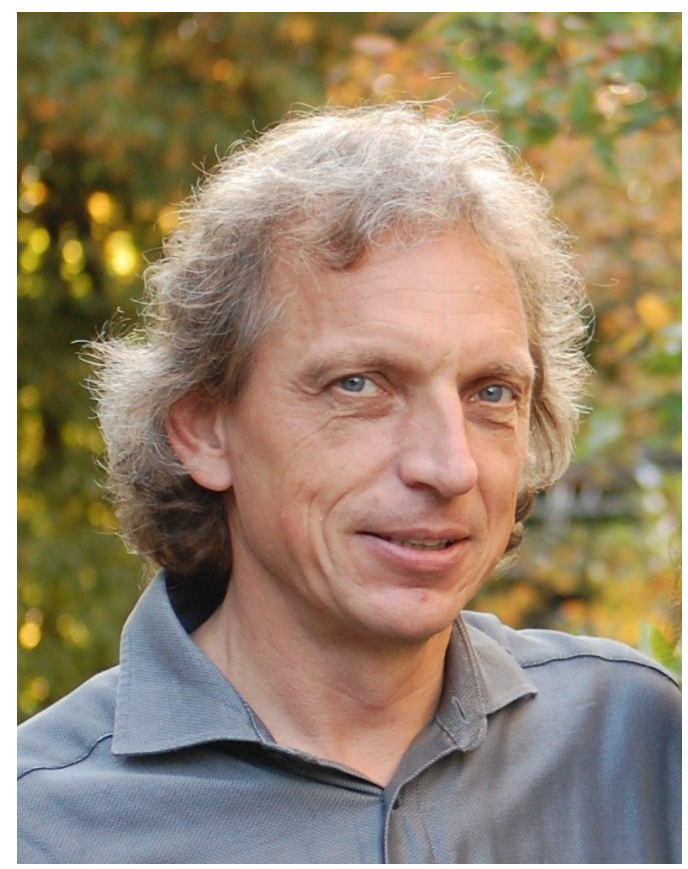

Christian von Ferber is internationally renowned for his works in statistical and condensed matter physics, complex systems and networks. His studies provided explanations for a whole range of phenomena in soft matter and complex systems, including scaling and multifractality in complex polymers, phase behavior of polyelectrolytes and surfactants, dynamics and vulnerability of complex networks. His engagement in preparation of young scientists and personal initiative in various forms of collaboration between different countries resulted in numerous successful projects that led both to advances of knowledge as well as creation of new forms of scientific collaborations.

Christian von Ferber was born on May 15, 1961 in Göttingen, Germany. He obtained a Master degree in Physics (Dipl. Phys.) in 1988 at the University of Bonn and performed PhD studies under the supervision of Prof. Lothar Schäfer in the University of Essen. There, the topic of his research was scaling properties of polymers of complex topology: polymer stars and networks. Applying a field theoretical renormalization group approach he obtained important results that led both to an accurate quantitative description and a deeper understanding of a whole range of phenomena where complex polymers are involved. In 1993 he defended his dissertation (Dr. rer. nat., summa cum laude). His subsequent research was performed in several leading scientific centers, including Tel Aviv University, where he spent two years as a Fellow of the prestigious Minerva Foundation, and the universities of Düsseldorf and Freiburg where he was a research fellow. There, he continued his research in the field of soft matter systems, enriching both the spectrum of objects of his studies as well as mastering state-of-the-art tools of analytical statistical physics and computer simulations. He was also Guest Professor at the University of Linz (Austria) and a Marie Curie Fellow at the University of Krakow (Poland). In 2003 he earned his Habilitation (Venia Legendi in Physics) at the University of Freiburg and held then Privatdozent positions in universities of 
Freiburg and Düsseldorf. In 2006 he started his work at the Applied Mathematics Research Centre in Coventry University (UK), first as a Senior Lecturer and afterwards as a Reader.

The scientific interests of Christian von Ferber mainly concern soft matter physics and complex systems. In the course of deep, systematic and vigorous research he became a leading expert in the fields of statistical physics, polymers and complex network theory. Subjects of his analyses range from complex polymers, colloids and disordered magnets to transportation networks. A particular feature of his scientific approach is the skillful application of a variety of modern analytical methods and combining them with computational tools. Scaling in complex polymer systems, multifractal phenomena, phase behavior of polyelectrolytes and surfactants, network dynamics and attack-vulnerability are only some of the phenomena which have become better understood due to his devoted work. His colleagues know and acknowledge his particular skills in capturing the main features of certain phenomena, suggesting appropriate models that explain these features and, finally, treating a problem both numerically and analytically.

Christian von Ferber has special status in two institutions: Coventry University in Great Britain, his home institution, and the Institute for Condensed Matter Physics (ICMP), in Ukraine, where he holds a title of Doctor Honoris Causa. His influence on Coventry was enormous - moving theoretical physics in the direction of statistical physics and initiating research in polymer physics and the science of complex networks. His successes in these directions encouraged a newly formed team there to follow these directions and his courage in initiating bilateral $\mathrm{PhD}$ studies opened the gates for dozens of students. These started as a PhD partnership (cotutelle de thèse) agreement between Coventry and Nancy universities and with a span of time transformed into International Doctoral College in Statistical Physics of Complex Systems, that joins now four institutions, along with the above two incorporating also ICMP and Leipzig University. It is in Coventry that he became a principal investigator and project leader of prestigious EU research projects, closely collaborating with research groups in France, Germany, Austria, Argentina, Brazil, the USA, India and other countries.

And it is in Lviv that he started joint research in polymer physics and complex system science as well as the organizational activities that last already almost for thirty years. He has long been a member of the editorial board of Condensed Matter Physics and lead polymer topics in this journal. In particular, he was the initiator and co-editor of several special issues of the journal, including the first collection of review articles on the physics of star polymers. Christian von Ferber has been involved in organisation of numerous conferences held by ICMP. His active cooperation contributed to the success and high reputation of these conferences. Much of his efforts were devoted to educating the younger generation of scientists, supervising Master and Doctoral theses from which particularly benefited young Ukrainian researchers. He taught special courses to students and graduate students of the Ivan Franko National University in Lviv and in the ICMP. On a larger scale, numerous students in different universities of Germany, Austria, Ukraine and the UK gratefully acknowledge his lecturing.

All who know Christian personally highly appreciate the charming features of his personality: his openness and readiness to help in different situations, his high-level of responsibility, his cordiality, his tact, his generosity, his sense of humor, his gentle smile, and his erudition. On the occasion his 60th birthday, the Editorial board of CMP, colleagues and friends warmly congratulate Christian von Ferber and thank him for all his devoted work not only for Physics but also for the community of Physicists!

Editorial board 